\title{
Attributable mortality of ICU acquired bloodstream infections: a propensity-score matched analysis
}

\author{
Nicolas Massart ${ }^{1,2,3} \mathbb{D} \cdot$ Guilhem Wattecamps $^{4} \cdot$ Mikael Moriconi $^{4} \cdot$ Pierre Fillatre $^{1}$ \\ Received: 16 July 2020 / Accepted: 1 March 2021 / Published online: 10 March 2021 \\ (C) The Author(s), under exclusive licence to Springer-Verlag GmbH Germany, part of Springer Nature 2021
}

\begin{abstract}
The mortality attributable to ICU-acquired bloodstream infection (BSI) differs between studies due to statistical methods used for cohort matching. Propensity-score matching has never been used to avoid eventual bias when studying BSI attributable mortality in the ICU. We conducted an observational prospective study over a 4-year period, on patients admitted for at least $48 \mathrm{~h}$ in 2 intensive care units. Based on risk factors for death in the ICU and for BSI, each patient with BSI was matched with 3 patients without BSI using propensity-score matching. We performed a competitive risk analysis to study BSI mortality attributable fraction. Of 2464 included patients, 71 (2.9\%) had a BSI. Propensity-score matching was highly effective and group characteristics were fully balanced. Crude mortality was $36.6 \%$ in patients with BSI and $21.6 \%$ in propensity-score matched patients $(p=0.018)$. Attributable mortality of BSI was $2.3 \%$ [1.2-4.0] and number needed to harm was 6.7. With Fine and Gray model, a higher risk for death was observed in patients with BSI than in propensity-score matched patients (sub distribution Hazard Ratio $(\mathrm{sdHR})=2.11 ; 95 \% \mathrm{CI}[1.32-3.37] p=0.002)$. Patients with BSI had a higher risk for death and BSI attributable mortality fraction was $2.3 \%$.
\end{abstract}

Keywords Critical care $\cdot$ Bacteremia $\cdot$ Mortality $\cdot$ Propensity score

\section{Introduction}

Critically ill patients in intensive care units (ICU) have a high prevalence of ICU acquired bloodstream infection (BSI) ranging from 5 to $7 \%$ (1-7). However, BSI-associated morbidity and mortality differ between studies, mainly due to potential cofounders or the methods used for statistical analysis (1-7). Since BSI usually develops in the most severly ill patients

Nicolas Massart and Guilhem Wattecamps contributed equally for this study and share first authorship.

Nicolas Massart

nicolasmassart@hotmail.fr

Service de Réanimation, $\mathrm{CH}$ de St BRIEUC, 10, rue Marcel Proust 22000 Saint-Brieuc France

2 Faculté de Médecine, Université Rennes 1, Biosit, F-35043 Rennes France

3 Service de maladie infectieuse et de réanimation médicale CHU de rennes, 2, rue Henri le Guilloux 35000 Rennes France

4 Service de Réanimation, CH de QUIMPER, 14bis Avenue Yves Thépot 29107 Quimper France
(2-6), BSI could be the cause of death but could also be a marker of the severity of the condition. Propensity score matching is appropriate for generation of control groups in observational studies and experts suggest its use to study hospital acquired infections and attributable mortality $(8,9)$. To our knowledge, such matching has been rarely used to study BSI. Therefore, we conducted an observational study with propensity-score matched analysis. The primary objective was to assess the attributable mortality fraction due to BSI during ICU stay.

\section{Material and methods}

\section{Setting and patients}

We conducted a prospective observational study in two French ICU with surgical and medical patients: a 14 bedsICU in Saint-Brieuc and a 12-beds ICU in Quimper which are part of tertiary hospitals with 700 and 600 acute-care beds respectively. All consecutive adults patients admitted from January 1, 2015, until December 31, 2018, with an ICU stay of 48 hours or more were included. Patients with liberty 
deprivation, pregnant women, and patients younger than 18 years old were excluded from the study. Patients or closest relative were informed of the prospective collection unless they disagreed of being included. The local ethics committee (Comité d'Éthique du CHU de Rennes) approved the study $\left(\mathrm{N}^{\circ} 18.53\right)$.

\section{Definition}

Infection was considered acquired in the ICU when it was diagnosed 48 hours after admission. BSI was defined according to the $\mathrm{CDC}(10,11)$ : for common skin contaminants, 2 positives blood cultures drawn on separate occasions were required and for other pathogens 1 positive blood culture. BSI that was thought to be seeded from another infection site was considered as secondary whereas BSI were considered as primary BSI when no simultaneous infection were found. Secondary BSI needed criteria for infection and same bacteria identified in specific localization culture specimens and in blood culture specimens. The diagnosis of pneumonia included both ventilator-associated pneumonia and ICU-acquired pneumonia that developed in non-ventilated patients and was made based on clinical signs (fever), radiological findings (new infiltrate on chest-X-ray or CT scan), and a positive microbiological culture of an endotracheal aspirate $\left(\geq 10^{6}\right.$ colony-forming units $/ \mathrm{mL}$ ) or a broncho-alveolar lavage ( $\geq 10^{4}$ colony-forming units $/ \mathrm{mL}$ ). Catheter-related bloodstream infection was considered in patients with BSI attributed to intravascular catheter (quantitative culture of the catheter tip or by differences in growth between catheter and peripheral venipuncture blood culture specimens). Urinary tract infection was considered in patients with catheter-associated symptomatic urinary tract infection, non-catheter related symptomatic urinary tract infection or asymptomatic bacteremic urinary tract infection. Tertiary peritonitis was considered in patients with intra-abdominal infection that persists or recurs $48 \mathrm{~h}$ following successful and adequate surgical source. Finally, surgical-site infection was considered in patients with fever and spontaneous or deliberate wound dehisces or other evidence of infection (on gross anatomical or histopathologic exam, or imaging test).

Baseline characteristics were registered at admission (simplified acute physiology score II, age, sex, diagnostic category [planned surgery/emergency surgery/medical/], admission origin [home, acute-care ward, long-term care facility, other intensive care unit], antimicrobial agents at admission, immunosuppression, and neutropenia). Other data was collected during the ICU stay (number of days at risk for BSI, intubation during the period at risk, use of a central catheter during the period at risk) or on the day of discharge for patients with no BSI during the ICU stay. The number of days at risk for BSI was the number of days in the ICU from the 48th hour of stay until ICU discharge for patients without BSI or until the first occurrence of BSI for patients with BSI.

\section{Primary and secondary endpoints}

The primary endpoint of the study was the fraction of ICU mortality attributable to BSI. The excess of ICU death due to BSI was the difference between the number of ICU death in the BSI and the propensity-score-matched groups. Attributable mortality fraction was defined as the percentage of ICU deaths caused by BSI and was calculated as the excess of death in the BSI group divided by the number of deaths during the study period (i.e., attributable mortality fraction $=[$ Mortality in BSI patients - Mortality in matched patients]/Mortality in the whole cohort). Then, based on the result of crude mortality in each groups, we calculated the number needed to harm. It corresponds to the number of patients that need to be exposed to BSI in order to result in a supplementary death (i.e., 1/[mortality rate in BSI group - mortality rate in control group]).

Association between source of BSI (i.e., primary/secondary) and death was secondary endpoints as were description of the micro-organisms causing BSI and their antimicrobial susceptibility.

\section{Statistical analysis}

Statistical analysis was performed with the statistical software R 3.4.3. The probability of developing a BSI was estimated using a multivariate logistic regression model that incorporated risk factors. First, to draw unbiased marginal estimates of exposure effect, a propensity-score matched analysis was performed. Propensity score was calculated for each patient and correspond to his probability to develop BSI and to die. As potential confounders, we included for propensity-score calculation all non-redundant variables associated with BSI (event) or ICU death (outcome) with $p$-value $\leq 0.20$ in the univariate analysis. Then, using the "MatchIt" package, a knearest neighbor algorithm was used for propensity-score matching with a 1:3 ratio: each patient with BSI was matched with 3 patients without BSI with the nearest propensity-score. Finally, a sensitive analysis was performed in the whole population with inverse probability weighting to generate a weighted cohort in which baseline characteristics distributions were independent of BSI exposure. It applied weights corresponding for "1/Propensity score" for patients exposed to BSI and "1/(1-Propensity score)" for control group.

Competitive risk analysis was used with ICU discharge and death in ICU being competing events. Using the "cmprsk" package we performed a Fine and Gray model to estimate sub-distribution hazard ratio (sdHR) of ICU death $(12,13)$. Therefore sdHR $>1$ indicates that those with exposure (BSI) will be seen to have a quicker time to death. Conversly, a sdHR $<1$ indicates a longer time before death for those exposed. 
Because BSI acquisition was considered as a transitions state from admission to discharge or death, a multistate approach was performed (13): patients with BSI were included first in the group without BSI until BSI onset, then censured from the group without BSI and included in the BSI group until discharge or death in the ICU.

Categorical variables were expressed as percentages and continuous variables as median and interquartile range (IQR). The chi-square test and Fisher exact test were used to compare categorical variables and the Man-Whitney U test for continuous variables. All tests were two-sided, and $p<0.05$ was considered statistically significant.

\section{Results}

\section{Population}

During the study period, 2464 patients stayed at least $48 \mathrm{~h}$ in our ICUs. Baseline characteristics of study patients are shown in Table 1. Median age was 64.9 years [55.2-73.4], and median SAPS II score was 44 [32-58]. Median ICU stay was 6 days [3-12], 1731 patients (70.3\%) were intubated during the period at risk for BSI, and 1621 had a central catheter $(65.8 \%)$. Four hundred fifty-nine patients $(18.6 \%)$ died in the ICU.

\section{Acquired infection}

Of the 2464 included patients, 231 (9.4\%) acquired 284 infections (10.8 acquired infections per 1000 patients-days) during their stay in ICU. Seventy-one had BSI (2.9\%) giving an incidence of 2.7/1000 patients-days and 4.2/1000 patients-days at risk, of whom 30 had primary BSI (1.2\%) and 41 had secondary BSI (1.7\%). Of notes, 185 patients had pneumonia (7.5\%), 16 had catheter related BSI $(0.6 \%), 9$ had tertiary peritonitis $(0.4 \%), 2$ had urinary tract infection $(0.1 \%)$ and 1 had surgical-site infection $(0.0 \%)$.

\section{Risk factors for BSI}

Factors independently associated with BSI were intubation $(\mathrm{OR}=2.72 ; 95 \% \mathrm{CI}[1.13-6.54] p=0.026])$ and use of a central catheter $(\mathrm{OR}=6.49 ; 95 \% \mathrm{CI}[2.28-18.47] p<0.001])$ during the period at risk for BSI.

High SAPS II score $(\mathrm{OR}=1.00 ; 95 \%$ CI $[0.99-1.01] p$ $=0.76])$ and number of day at risk for BSI $(\mathrm{OR}=1.00 ; 95 \%$

Table 1 Baseline characteristics and outcome of study patients

\begin{tabular}{|c|c|c|c|c|c|c|c|c|}
\hline \multirow{3}{*}{ Age, years, median [IQR] } & \multirow{2}{*}{\multicolumn{2}{|c|}{$\frac{\text { All patients }}{n=2464}$}} & \multirow{2}{*}{\multicolumn{2}{|c|}{$\begin{array}{l}\text { Patients with BSI } \\
n=71\end{array}$}} & \multirow{2}{*}{\multicolumn{2}{|c|}{$\begin{array}{l}\text { Matched patients } \\
n=213\end{array}$}} & \multirow{3}{*}{$\begin{array}{l}p \text {-value* } \\
0.85\end{array}$} & \multirow{3}{*}{$\begin{array}{l}\text { Standardized } \\
\text { mean difference }\end{array}$} \\
\hline & & & & & & & & \\
\hline & 64.9 & {$[55.2-73.4]$} & 64.8 & {$[53.1-70.4]$} & 63.7 & {$[55.2-62.0]$} & & \\
\hline SAPS II, median [IQR] & 44 & {$[32-58]$} & 53 & {$[34-66]$} & 48 & {$[37-64]$} & 0.80 & 1.00 \\
\hline Male - no. $(\%)$ & 1598 & $(64.9)$ & 49 & $(69.0)$ & 147 & $(69.0)$ & 1 & 0.00 \\
\hline \multicolumn{9}{|l|}{ Localization before admission } \\
\hline Acute care ward - no. $(\%)$ & 914 & $(37.1)$ & 31 & $(43.7)$ & 87 & $(40.9)$ & 0.78 & 0.03 \\
\hline Home - no. $(\%)$ & 1439 & $(58.4)$ & 36 & $(50.7)$ & 111 & $(52.1)$ & 0.94 & 0.01 \\
\hline Long-term care facility- no. (\%) & 31 & $(1.3)$ & 2 & $(2.8)$ & 9 & $(4.2)$ & 0.86 & 0.01 \\
\hline Other ICU - no. $(\%)$ & 53 & $(2.2)$ & 1 & (1.4) & 4 & (1.9) & 0.75 & 0.00 \\
\hline \multicolumn{9}{|l|}{ Reason for admission } \\
\hline Trauma - no. $(\%)$ & 162 & $(65.8)$ & 4 & $(5.6)$ & 16 & $(7.5)$ & 0.79 & 0.02 \\
\hline Surgical - no. (\%) & 496 & $(20.1)$ & 17 & $(23.9)$ & 54 & $(25.4)$ & 0.94 & 0.01 \\
\hline Medical - no. (\%) & 1968 & $(79.9)$ & 54 & $(76.1)$ & 159 & $(74.6)$ & 0.94 & 0.01 \\
\hline Antibiotic before admission - no. (\%) & 1434 & $(58.2)$ & 42 & $(59.2)$ & 136 & $(63.9)$ & 0.57 & 0.00 \\
\hline Immunosuppresion - no. (\%) & 140 & $(5.7)$ & 4 & $(5.6)$ & 10 & $(4.7)$ & 1 & 0.00 \\
\hline Neutropenia $(<500 / \mathrm{mL})-$ no. $(\%)$ & 31 & $(1.3)$ & 1 & (1.4) & 2 & $(0.9)$ & 1 & 0.00 \\
\hline Intubation during period at risk for BSI - no. $(\%)$ & 1731 & $(70.3)$ & 65 & $(91.6)$ & 196 & $(92.0)$ & 1 & 0.00 \\
\hline Catheter during period at risk for BSI - no. $(\%)$ & 1621 & $(65.8)$ & 67 & $(94.4)$ & 202 & $(94.8)$ & 1 & 0.00 \\
\hline Number of days at risk for BSI, days, median [IQR] & 4 & {$[1-9]$} & 6 & {$[3-12]$} & 6 & {$[2-11]$} & 0.64 & 0.06 \\
\hline Lenght of stay in ICU, days, median [IQR] & 6 & {$[3-12]$} & 18 & [13-44] & 8 & {$[5-14]$} & $<0.001$ & \\
\hline Death in ICU - no. $(\%)$ & 459 & $(18.6)$ & 26 & (36.6) & 46 & (21.6) & 0.018 & \\
\hline
\end{tabular}

$I C U$ intensive care unit. *For comparison between patients with BSI and matched patients 
CI $[0.97-1.02] p=0.65]$ ) were not associated with BSI (Table 2).

\section{Risk factors for death in ICU}

Factors independently associated with death in the ICU were a higher age $(\mathrm{OR}=1.02 ; 95 \% \mathrm{CI}[1.01-1.03] p<0.001])$, higher SAPS II score $(\mathrm{OR}=1.02 ; 95 \%$ CI $[1.02-1.03] p<0.001])$, surgical patients $(\mathrm{OR}=0.50 ; 95 \% \mathrm{CI}[0.36-0.68] p<0.001])$, antibiotics prior to ICU admission $(\mathrm{OR}=0.78$; $95 \% \mathrm{CI}[0.62-$ 0.97] $p=0.029]$ ), immunosuppression ( $\mathrm{OR}=1.70 ; 95 \% \mathrm{CI}$ $[1.11-2.60] p=0.013])$, intubation $(\mathrm{OR}=3.60$; 95\% CI [2.475.27] $p<0.001])$, and use of a central catheter $(\mathrm{OR}=2.13$; 95\% CI [1.55-2.92] $p<0.001]$ ) during the period at risk for acquired BSI. The acquisition of an infection during ICU stay was not associated with an increased risk for death in our study (OR $=0.94 ; 95 \%$ CI $[0.67-1.32] p=0.71)$. However, acquired BSI were independently associated with death $(\mathrm{OR}=$ $1.88 ; 95 \% \mathrm{CI}[1.10-3.23] p=0.021)$, especially for secondary BSI $(\mathrm{OR}=3.23 ; 95 \% \mathrm{CI}[1.60-6.51] p=0.001)$ while primary BSI were not statistically associated with death $(\mathrm{OR}=0.80$; 95\% CI [0.31-2.07] $p=0.65$ ) (Table 3 ).

\section{BSI groups and propensity-score analysis}

In patients with BSI, median age was 64.8 years [53.1-70.4] and median SAPS II score was 53 [34-66]. They were more often intubated during the period at risk for BSI $(91.6 \%$ vs
$70.3 \%)$ and more frequently had central catheter $(94.4 \%$ vs $65.8 \%$ ) than the overall cohort. For propensity-score calculation, every variable associated with BSI or death in the ICU with a $p$-value $<0.2$ was included in the analysis (i.e., higher SAPS II score, higher age, sex, admission for trauma, admission from another ICU, admission from a long-term care facility, surgical patients, immunosuppression, neutropenia $(<500 /$ $\mathrm{mL}$ ), antibiotics prior to ICU admission, number of day at risk for BSI, intubation and use of a central catheter during the period at risk for BSI). Based on their propensity-score, the 71 patients with BSI were matched with 213 patients without BSI with the nearest propensity-score (supplementary Fig. 1). Baseline characteristics of the 2 groups are reported in Table 1 and were balanced (Table 1). Patients with BSI had a significantly longer stay than propensity-score matched patients (18 days [13-44] versus 8 days [5-14], $p<0.001)$.

\section{Attributable mortality}

In the BSI group, 26 patients (36.7\%) died while 46 patients $(21.6 \%)$ died in the control group ( $p=0.018)$ (Fig. 1). Based on this results, excess of death due to BSI in the BSI group was $15.1 \%$ (approximatively 11 patients) and number needed to harm was 6.7 (i.e., there were 1 supplementary death per 6.7 patients exposed to BSI). Therefore, the mortality attributable fraction of BSI in the overall population was $2.3 \%$ [1.2-4.0] (i.e., 2.3\% of the ICU deaths were related to BSI). With Fine and Gray model, BSI patients had a higher probability to die in

Table 2 Risk factors for BSI

\begin{tabular}{|c|c|c|c|c|c|c|}
\hline & \multicolumn{3}{|c|}{ Univariate analysis } & \multicolumn{3}{|c|}{ Multivariate analysis } \\
\hline & OR & $95 \% \mathrm{CI}$ & $p$-value & OR & $95 \% \mathrm{CI}$ & $p$-value \\
\hline Age, per supplementary year & 0.99 & {$[0.98-1.01]$} & 0.49 & & & \\
\hline $\begin{array}{l}\text { Simplified acute physiology score II, per } \\
\text { one point increment }\end{array}$ & 1.00 & {$[1.00-1.01]$} & 0.14 & 1.00 & {$[0.99-1.01]$} & 0.76 \\
\hline Male & 1.21 & {$[0.73-2.02]$} & 0.46 & & & \\
\hline Admission from a long-term care facility & 2.36 & {$[0.55-10.10]$} & 0.25 & & & \\
\hline Admission from an acute care ward & 1.33 & {$[0.82-2.13]$} & 0.25 & & & \\
\hline Admission from Home & 0.73 & {$[0.45-1.16]$} & 0.18 & 0.77 & {$[0.48-1.24]$} & 0.28 \\
\hline Admission from an other ICU & 0.64 & {$[0.09-4.72]$} & 0.66 & & & \\
\hline Admission for trauma & 0.84 & {$[0.30-2.35]$} & 0.75 & & & \\
\hline Surgical reason for admission & 1.26 & {$[0.72-2.19]$} & 0.42 & & & \\
\hline Antibiotic before admission & 1.04 & {$[0.64-1.68]$} & 0.87 & & & \\
\hline Immunosuppresion & 0.99 & {$[0.36-2.76]$} & 0.99 & & & \\
\hline Neutropenia $(<500 / \mathrm{mL})$ & 1.13 & {$[0.15-8.37]$} & 0.91 & & & \\
\hline Intubation during period at risk for BSI & 4.73 & [2.04-10.96] & $<0.001$ & 2.72 & {$[1.13-6.54]$} & 0.026 \\
\hline $\begin{array}{l}\text { Central catheter during period at risk for } \\
\text { BSI }\end{array}$ & 9.04 & [3.29-24.89] & $<0.001$ & 6.49 & {$[2.28-18.47]$} & $<0.001$ \\
\hline $\begin{array}{l}\text { Number of day at risk for BSI, per } \\
\text { supplementary days }\end{array}$ & 1.02 & {$[1.00-1.04]$} & 0.13 & 1.00 & {$[0.97-1.02]$} & 0.65 \\
\hline
\end{tabular}

$I C U$ intensive care unit. $B S I$ bloodstream infection 
Table 3 Risk factors for death in the ICU

\begin{tabular}{|c|c|c|c|c|c|c|}
\hline & \multicolumn{3}{|c|}{ Univariate analysis } & \multicolumn{3}{|c|}{ Multivariate analysis } \\
\hline & OR & $95 \% \mathrm{CI}$ & $p$-value & OR & $95 \% \mathrm{CI}$ & $p$-value \\
\hline Age, per supplementary year & 1.0 & $1.02-1.03$ & $<0.001$ & 1.02 & $1.02-1.03$ & $<0.001$ \\
\hline $\begin{array}{l}\text { Simplified acute physiology score II, per one } \\
\text { point increment }\end{array}$ & 1.04 & $1.03-1.05$ & $<0.001$ & 1.02 & $1.02-1.03$ & $<0.001$ \\
\hline Male & 1.34 & $1.08-1.67$ & 0.009 & 1.17 & $0.93-1.49$ & 0.19 \\
\hline Admission from a long-term care facility & 2.10 & $0.98-4.50$ & 0.055 & 1.96 & $0.84-4.56$ & 0.12 \\
\hline Admission from an acute care ward & 1.03 & $0.84-1.27$ & 0.76 & & & \\
\hline Admission from Home & 0.97 & $0.79-1.20$ & 0.81 & & & \\
\hline Admission from an other ICU & 0.55 & $0.23-1.30$ & 0.17 & 0.92 & $0.38-2.24$ & 0.85 \\
\hline Admission for trauma & 0.60 & $0.37-0.97$ & 0.035 & 0.89 & $0.53-1.50$ & 0.67 \\
\hline Surgical reason for admission & 0.55 & $0.41-0.73$ & $<0.001$ & 0.50 & $0.36-0.69$ & $<0.001$ \\
\hline Antibiotic before admission & 0.84 & $0.69-1.04$ & 0.11 & 0.78 & $0.62-0.97$ & 0.029 \\
\hline Immunosuppresion & 1.82 & $1.24-2.66$ & 0.002 & 1.70 & $1.11-2.60$ & 0.013 \\
\hline Neutropenia $(<500 / \mathrm{mL})$ & 1.80 & $0.82-3.94$ & 0.14 & 1.22 & $0.53-2.83$ & 0.64 \\
\hline Intubation during period at risk for BSI & 5.88 & $4.16-8.30$ & $<0.001$ & 3.60 & $2.47-5.27$ & $<0.001$ \\
\hline Catheter during period at risk for BSI & 4.17 & $3.14-5.54$ & $<0.001$ & 2.13 & $1.55-2.92$ & $<0.001$ \\
\hline $\begin{array}{l}\text { Number of day at risk for BSI, per } \\
\text { supplementary days } \\
\text { Infection acquired in the ICU* }\end{array}$ & 1.01 & $1.00-1.02$ & 0.22 & & & \\
\hline Any acquired infection & 1.49 & $1.09-2.05$ & 0.014 & 0.94 & $0.67-1.32$ & 0.71 \\
\hline BSI & 2.62 & $1.60-4.29$ & $<0.001$ & 1.88 & $1.10-3.23$ & 0.021 \\
\hline Primary BSI & 1.09 & $0.44-2.69$ & 0.85 & 0.80 & $0.31-2.07$ & 0.65 \\
\hline Secondary BSI & 4.30 & $2.31-8.01$ & $<0.001$ & 3.23 & $1.60-6.51$ & 0.001 \\
\hline
\end{tabular}

$I C U$ intensive care unit. $B S I$ bloodstream infection

*Each type of infection was tested alone in the multivariable analysis the ICU than matched patients $(\mathrm{sdHR}=2.11 ; 95 \% \mathrm{CI}[1.32$ 3.37] $p=0.002$ ).

Similarly, in the inverse probability of BSI weighting analysis including the full cohort, patients with BSI had a higher hazard of death than patients without BSI (cox model: HR = $1.90 ; 95 \%$ CI $[1.69-2.13] p<0.001)$.

\section{Microorganisms and antimicrobial susceptibility}

Gram-negative bacilli were found in 49 samples (59.4\% of the micro-organisms), and Enterobacteriacae were the main cause of blood-stream infections (40 isolates; $44.9 \%$ of the micro-organisms). There were 3 (7.5\%) extended-spectrumbeta-lactamase producing Enterobacteriacae isolates. Pseudomonas aeruginosas was isolated from 9 blood cultures (10.1\%), and 3 isolates $(33.4 \%)$ were resistant to ceftazidim, piperacillin-tazobactam and imipenem. All Gram-negative bacilli were susceptible to amikacin (Table 4).

Gram-positive cocci were found in 28 blood cultures (31.5\%), with 13 Staphylococcus aureus (14.6\%). Only 2 isolates $(15.4 \%)$ were resistant to methicillin. Of 7 isolated coagulase negative staphylococci, 2 were susceptible to methicillin
$(66.3 \%)$. There were 6 isolates of enterococci, 1 was an Enterococcus faecium with natural ampicillin resistance. No vancomycin-resistant enterococci and no ampicillin-resistant streptococci were identified.

Yeasts were isolated from 8 patients $(9.0 \%$ of the microorganisms). All were susceptible to fluconazole.

Three isolated anaerobic bacteria (3.4\% of isolated microorganisms) were susceptible to penicillin in combination with beta-lactamase inhibitors.

\section{Discussion}

Mortality in patients with BSI varies between studies (2-6). In our study, mortality in the BSI group was $36.6 \%$ which is lower than in older studies $(2,4)$. As previously found, BSI was independently associated with a 2- to 3 -fold increase in mortality compared with patients without BSI suggesting that this association has remained true over time (2-6).

When investigating the outcomes of ICU acquired infection, only observational studies are feasible. Direct comparison between patients with and without BSI may lead to bias since 
Fig. 1 BSI group
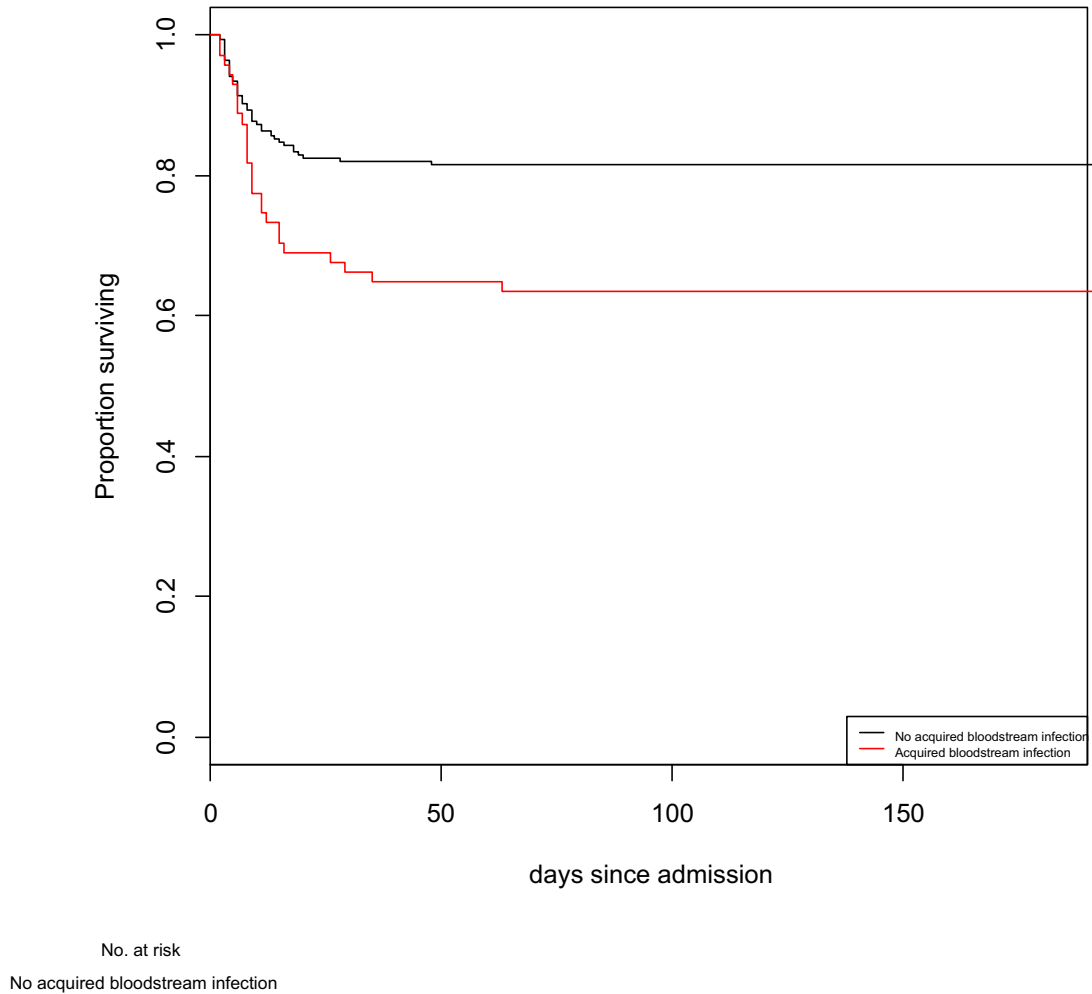

Acquired bloodstream infection

days since admission

$(8,9)$. Although matching process results in well-balanced

baseline characteristics differ. In order to address this problem, length of exposure to risk of infection may be considered (9), as it has been done in previous publications, with time-dependent analysis (2) or with matching characteristics (6) or with logistic regression (3). As propensity-score matched analysis allows unbiased marginal estimates of exposure effects, it is the best method to create a proper control group in observational studies characteristics, length of stay differed significantly between the two groups. Of note, length of exposure to risk of BSI was used for propensity-score matching, which can explain this difference. Although median duration of exposure to the risk of BSI (number of days without BSI) did not differ between groups, 94 of the 213 matched patients (44\%) were discharge

Table 4 Microorganisms isolated from bloodstream infections and their antibiotic susceptibility

\begin{tabular}{|c|c|c|c|c|c|c|c|c|}
\hline $\begin{array}{l}\text { Micro-organism* no. susceptible / } \\
\text { no. tested }(\%)\end{array}$ & $\begin{array}{l}\text { Number of } \\
\text { isolates }\end{array}$ & Ampicillin & Methicillin & Ceftazidime & $\begin{array}{l}\text { Piperacillin- } \\
\text { tazobactam }\end{array}$ & Imipenem & Amikacin & Fluconazole \\
\hline Enterobacteriacae & 40 & - & - & - & $31 / 33(93.9)$ & $\begin{array}{l}32 / 33 \\
\quad(97.0)\end{array}$ & $\begin{array}{l}33 / 33 \\
\quad(100)\end{array}$ & - \\
\hline Staphylococcus. aureus & 13 & & $\begin{array}{l}11 / 13 \\
(84.6)\end{array}$ & - & - & - & - & - \\
\hline Pseudomonas aeruginosa & 9 & - & - & 6/9 (66.6) & $6 / 9(66.6)$ & $6 / 9(66.6)$ & 9/9 (100) & - \\
\hline Coagulase Negative Staphylococcus & 7 & - & $2 / 6(33.3)$ & - & - & - & - & - \\
\hline Enterococcus $S p^{*}$ & 6 & $5 / 6(83.3)$ & - & - & - & - & - & - \\
\hline Streptococcus Sp. & 2 & $2 / 2(100)$ & - & - & - & - & - & - \\
\hline Anaerobes & 3 & - & - & - & $3 / 3(100)$ & - & - & - \\
\hline Haemophilus Sp. & 1 & 1/1 (100) & - & - & - & - & - & - \\
\hline Candida Sp. & 8 & - & - & - & - & - & - & 8/8 (100) \\
\hline Total & 89 & 8/9 (88.9) & $\begin{array}{l}13 / 19 \\
(68.4)\end{array}$ & 6/9 (66.6) & 40/45 (88.9) & $\begin{array}{l}38 / 42 \\
\quad(90.5)\end{array}$ & $\begin{array}{l}42 / 42 \\
\quad(100)\end{array}$ & 8/8 (100) \\
\hline
\end{tabular}

*No glycopeptid-resistant enterococcus were found 
prior to the time corresponding-case patients acquired BSI. Because this could be addressed as a matching bias, we performed a sensitive analysis with inverse probability weighting in the full cohort; which confirmed that BSI was associated with a higher hazard of death.

In our study, mortality attributable fraction to BSI calculated with a propensity-score matched analysis was $2.3 \%$, lower than in previously published studies (2-6). Although they reported a higher rate of BSI $(5.2 \%)$ than that in our study, Prowle et al. found that BSI accounted for $1 \%$ of deaths (2). Nevertheless, BSI mortality attributable fraction in our study and in previously published studies (2-6), is relatively high $(2.3 \%)$ with regard to the rate of ICU acquired BSI (2.9\%). Surprisingly, only secondary BSI were independently associated with death whereas primary BSI were not, as foundin previous publications. Assessing outcome of BSI with respect for its source, Renaud et al. concluded that risk of mortality associated with primary and catheter-related bacteremia appears much lower than that of secondary bacteremia with an excess mortality of $20 \%$ and $55 \%$ respectively (14). Whereas Di Giovine et al. found no difference in the crude mortality between patients with primary BSI and matched patients (15).

Our microbiological findings are similar to findings in other French ICUs (16) and to the EUROBACT international cohort study by Tabah and al. (17). In an Italian ICU (18), the authors found a pronounced shift in the main causative micro-organsims, from Staphylococci to Enterobacteriacae between 2000 and 2012. This could be due to more stringent implementation of hand-washing policies, leading to a major decrease of bacteremia falsely attributed to coagulase negative staphylococci (19). .Of note, the present study was conducted in ICUs with low resistance prevalence rate (20) and BSI attributable mortality may differ with results observed in ICUs with other resistance prevalence.

The main strength of our study is the comparison with a propensity-score matched control population, reducing potential bias. To our knowledge, this method has been rarely used in order to assess BSI attributable mortality. A PubMed search was performed with the following criteria: "Bacteremia"[Mesh]) AND "Intensive Care Units"[Mesh]) AND "Cross Infection"[Mesh]) AND "Mortality"[Mesh]. Out of the 91 articles identified, only 23 studied specifically attributable mortality of ICU-acquired BSI. One only used a propensity score matching method to pair the exposed patient to the non-exposed patients in a large population of European ICU-patients in between 2008 and 2012 (21). They found a 5\% attributable mortality of ICU-acquired BSI.

Our study has many limitations. First, we did not acknowledge immortal time bias. Patient with BSI may have an increased risk for death in the ICU due to a longer ICU stay than control patients. However this bias was reduced thanks to matching including length of exposure to the risk of BSI. Then, all variables that impact prognosis have not been assessed $(2,22,23)$. For example, we did not consider the time between suspicions of BSI and start of antimicrobial treatment. We did not study the impact of inappropriate antimicrobial. However, our work was conducted to evaluate the mortality attributable to BSI in our ICU and not specifically the outcome in relation to previous and subsequent exposure.

\section{Conclusion}

In our prospective observational study, patients with BSI had a higher risk for death and mortality attributable to BSI estimated with a propensity-score matched analysis was $2.3 \%$. Our results can be used to design future clinical trials.

Supplementary Information The online version contains supplementary material available at https://doi.org/10.1007/s10096-021-04215-4.

Data availability Data will be made available on appropriate demand.

\section{Declarations}

Ethics approval This study respect de French reference method MR003. Patients or closest relative were informed of the prospective collection of the data and only those who provided informed consent were enrolled. The local ethics committee (Comité d'Éthique du CHU de Rennes) approved the study $\left(\mathrm{N}^{\circ} 18.53\right)$.

Conflict of interest The authors declare that they have no conflict of interest.

\section{References}

1. Vincent J, Rello J, Marshall J et al (2009) International Study of the Prevalence and Outcomes of Infection in Intensive Care Units. JAMA 302(21):2323-2329

2. Prowle JR, Echeverri JE, Ligabo EV, Sherry N, Taori GC, Crozier $\mathrm{TM}$ et al (2011) Acquired bloodstream infection in the intensive care unit: incidence and attributable mortality. Crit Care 15(2):R100

3. Adrie C, Garrouste-Orgeas M, Ibn Essaied W, Schwebel C, Darmon M, Mourvillier B et al (2017) Attributable mortality of ICU-acquired bloodstream infections: impact of the source, causative micro-organism, resistance profile and antimicrobial therapy. J Inf Secur 74:131-141

4. Garrouste-Orgeas M, Timsit JF, Tafflet M, Misset B, Zahar JR, Soufir L et al (2006) Excess risk of death from intensive care unit-acquired nosocomial bloodstream infections: a reappraisal. Clin Infect Dis 42:1118-1126

5. Higuera F, Rangel-Frausto MS, Rosenthal VD, Soto JM, Castañon J, Franco G, Tabal-Galan N, Ruiz J, Duarte P, Graves N (2007) Attributable cost and length of stay for patients with central venous catheter-associated bloodstream infection in Mexico City intensive care units: a prospective, matched analysis. Infect Control Hosp Epidemiol 28:31-35

6. Thompson DS (2008) Estimates of the rate of acquisition of bacteraemia and associated excess mortality in a general intensive care unit: a 10 year study. J Hosp Infect 69:56-61 
7. Blot SI, Vandewoude KH, Colardyn FA (2003) Evaluation of outcome in critically ill patients with nosocomial enterobacter bacteremia: results of a matched cohort study. Chest. 123:1208-1213

8. Rosenbaum PRRD (1983) The central role of the propensity score in observational studies for causal effects. Biometrika. 70:41-55

9. Timsit JF, Zahar JR, Chevret S (2011) Attributable mortality of ventilator-associated pneumonia. Curr Opin Crit Care 17(5):464 471

10. Garner JS, Jarvis WR, Emori TG, Horan TC, Hughes JM (1988) CDC definitions for nosocomial infections, 1988. Am J Infect Control 16(3): 128-140

11. Pneumonia (Ventilator-associated [VAP] and non-ventilatorassociated Pneumonia [PNEU]) Event; Bloodstream Infection Event (Central Line-Associated Bloodstream Infection and Noncentral Line Associated Bloodstream Infection); Urinary Tract Infection (Catheter-Associated Urinary Tract Infection [CAUTI] and Non-Catheter-Associated Urinary Tract Infection [UTI]) Events; Surgical Site Infection (SSI) Event available at https:// www.cdc.gov/nhsn/PDFs/pscManual/6pscVAPcurrent.pdf and https://www.cdc.gov/nhsn/PDFs/pscManual/4PSC CLABScurrent.pdf and https://www.cdc.gov/nhsn/PDFs/ pscManual/7pscCAUTIcurrent.pdf and https://www.cdc.gov/ nhsn/pdfs/pscmanual/9pscssicurrent.pdf. Accessed June 2020

12. Lau B, Cole SR, Gange SJ (2009) Competing risk regression models for epidemiologic data. Am J Epidemiol 170(2):244-256. https://doi.org/10.1093/aje/kwp107

13. Putter H, Fiocco M, Geskus RB (2007) Tutorial in biostatistics: competing risks and multi-state models. Stat Med 26(11):23892430. https://doi.org/10.1002/sim.2712

14. Renaud B, Brun-Buisson C (2001) Outcomes of primary and catheter-related bacteremia. A cohort and case-control study in critically ill patients. Am J Respir Crit Care Med 163:1584-1590

15. DiGiovine B, Chenoweth C, Watts C, Higgins M (2011) The attributable mortality and costs of primary nosocomial bloodstream infections in the intensive care unit. J Antimicrob Chemother 66: 1948-1953

16. Lepape A, Machut A, Savey A (2018) Réseau national Réa-Raisin de surveillance des infections acquises en réanimation adulte Méthodes et principaux résultats. Méd Intensive Réanim 27(3): 197-203
17. Tabah A, Koulenti D, Laupland K, Misset B, Valles J (2012) Bruzzi de Carvalho F, et al. Characteristics and determinants of outcome of hospital-acquired bloodstream infections in intensive care units: the EUROBACT International Cohort Study. Intensive Care Med 38(12):1930-1945

18. de Santis V, Gresoiu M, Corona AM, Wilson APR, Singer M (2015) Bacteraemia incidence, causative organisms and resistance patterns, antibiotic strategies and outcomes in a single university hospital ICU: continuing improvement between 2000 and 2013. J Antimicrob Chemother 70(1):273-278

19. Sharek PJ, Benitz WE, Abel NJ, Freeburn MJ, Mayer ML, Bergman DA (2002) Effect of an Evidence-Based Hand Washing Policy on Hand Washing Rates and False-Positive Coagulase Negative Staphylococcus Blood and Cerebrospinal Fluid Culture Rates in a Level III NICU. J Perinatol 22(2):137-143

20. Massart N, Camus C, Benezit F et al (2020) Incidence and risk factors for acquired colonization and infection due to extendedspectrum beta-lactamase-producing Gram-negative bacilli: a retrospective analysis in three ICUs with low multidrug resistance rate. Eur J Clin Microbiol Infect Dis 39:889. https://doi.org/10.1007/ s10096-019-03800-y

21. European Centre for Disease Prevention and Control. Incidence and attributable mortality ofhealthcare-associated infections in intensive care units in Europe, 2008-2012. Stockholm: ECDC; 2018

22. Kang C-I, Kim S-H, Park WB, Lee K-D, Kim H-B, Kim E-C, Myoung-don O, Choe K-W (2005) Bloodstream Infections Caused by Antibiotic-Resistant Gram-Negative Bacilli: Risk Factors for Mortality and Impact of Inappropriate Initial Antimicrobial Therapy on Outcome. Antimicrob Agents Chemother 49(2):760-766

23. Girometti N, Lewis RE, Giannella M, Ambretti S, Bartoletti M, Tedeschi $\mathrm{S}$ et al (2014) Klebsiella pneumoniae Bloodstream Infection: Epidemiology and Impact of Inappropriate. Empirical Therapy. Medicine (Baltimore) 93(17):298-309

Publisher's note Springer Nature remains neutral with regard to jurisdictional claims in published maps and institutional affiliations. 